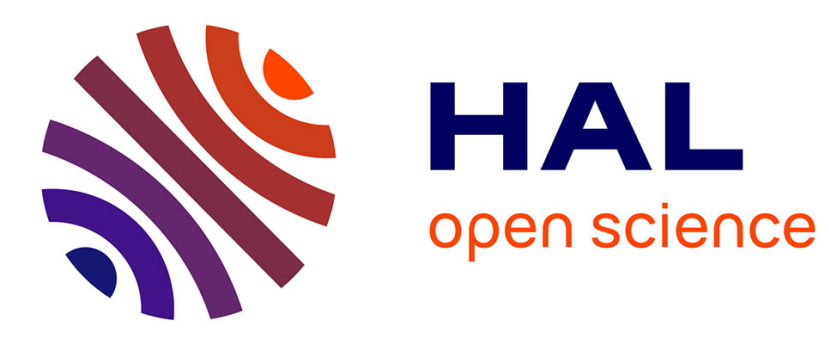

\title{
Non-Cooperative Routing in Loss Networks
}

\author{
Eitan Altman, Rachid El-Azouzi, Vyacheslav Abramov
}

\section{To cite this version:}

Eitan Altman, Rachid El-Azouzi, Vyacheslav Abramov. Non-Cooperative Routing in Loss Networks.

RR-4405, INRIA. 2002. inria-00072183

\section{HAL Id: inria-00072183 \\ https://hal.inria.fr/inria-00072183}

Submitted on 23 May 2006

HAL is a multi-disciplinary open access archive for the deposit and dissemination of scientific research documents, whether they are published or not. The documents may come from teaching and research institutions in France or abroad, or from public or private research centers.
L'archive ouverte pluridisciplinaire HAL, est destinée au dépôt et à la diffusion de documents scientifiques de niveau recherche, publiés ou non, émanant des établissements d'enseignement et de recherche français ou étrangers, des laboratoires publics ou privés. 


\section{NON-COOPERATIVE ROUTING IN LOSS NETWORKS}

Eitan ALTMAN — Rachid EL AZOUZI — Vyacheslav ABRAMOV

\section{$\mathbf{N}^{\circ} \mathbf{4 4 0 5}$}

MARS 2002

THÈME 1 



\title{
NON-COOPERATIVE ROUTING IN LOSS NETWORKS
}

\author{
Eitan ALTMAN $^{*}$, Rachid EL AZOUZI ${ }^{\dagger}$, Vyacheslav ABRAMOV ${ }^{\ddagger}$ \\ Thème 1 - Réseaux et systèmes \\ Projet MISTRAL
}

Rapport de recherche $\mathrm{n}^{\circ} 4405$ - MARS $2002-20$ pages

\begin{abstract}
The paper studies routing in loss networks in the framework of a non-cooperative game with selfish users. Two solution concepts are considered: the Nash equilibrium, corresponding to the case of a finite number of agents (such as service providers) that take routing decisions, and the Wardrop equilibrium, in which routing decisions are taken by a very large number of individual users. We show that these equilibria do not fall into the standard frameworks of non-cooperative routing games. As a result, we show that uniqueness of equilibria or even of utilizations at equilibria may fail even in the case of simple topology of parallel links. However, we show that some of the problems disappear in the case in which the bandwidth required by of all connections is the same. For the special case of a parallel link topology, we obtain some surprisingly simple way of solving the equilibrium for both cases of Wardrop as well as Nash equilibrium.
\end{abstract}

Key-words: Loss networks, Game theory, Nash equilibrium, Wardrop equilibrium .

* INRIA B.P 93, 2004 Route des Lucioles 06902 Sophia Antipolis Cedex France

$\dagger$ INRIA B.P 93, 2004 Route des Lucioles 06902 Sophia Antipolis Cedex France

$\ddagger$ Email: vyachesl@inter.net.il 


\section{Routage non-coopératif dans les réseaux à pertes}

Résumé : Nous étudions dans cet article le routage dans des réseaux à pertes dans le cadre de jeux non-coopératifs avec des usagers individualistes. Deux concepts de solutions sont considérés: l'équilibre de Nash, qui correspond au cas d'un nombre fini d'agents (tel que des fournisseurs de service) qui prennent des action de routage, et l'équilibre de Wardrop, où les décisions de routages sont prises par un très grand nombre d'usagers individuels. Nous montrons que ces équilibres ne tombent pas dans les cadres standards de jeux noncoopératifs de routage. Par conséquent, nous montrons que l'unicité de l'équilibre n'est pas satisfaite même dans la topologie la plus simple de liens parallèles, et l'utilisation des liens n'est pas unique non plus. Cependant, nous montrons que ce problème dispara^quand les bande passantes demandées par les connexions sont les mêmes. Pour le cas des liens

parallèles, nous obtenons une méthode simple pour résoudre les problèmes de l'équilibre de Nash et de Wardrop.

Mots-clés : Réseaux à pertes, théorie des jeux, équilibre de Nash, équilibre de Wardrop. 


\section{Introduction}

Non-cooperative routing has long been studied both in the framework of road-traffic as well as in the framework of telecommunication networks. Such frameworks allow to model the flow configuration that results in networks in which routing decisions are made in a noncooperative and distributed manner between the users. In case of a very large number of users, the resulting flow configuration corresponds to the so called Wardrop equilibrium [18] which is based on two principles: (i) all routes actually used between a source and a destination have the same delay (cost), and (ii) this delay (or cost) is no larger than that along any other route between that source-destination pair. In the case of a finite (not very large) number of agents, the resulting flow configuration corresponds to the so called Nash equilibrium [2] defined as a situation in which no agent has an incentive to deviate unilaterally. Although Wardrop equilibrium has mainly been used in the road traffic context, it has also been defined independently in other areas: in economy (see [15]) and in telecommunications [5] in the framework of Ad Hoc Networks. The Nash equilibrium has been extensively used in telecommunications, see e.g. $[13,10]$. When attempting to use the classical results concerning Wardrop equilibrium or Nash equilibrium for non-cooperative routing games for analyzing general loss networks, one is faced with the following problems.

- The theory that has been developed for routing games assumes that costs are sum of link costs. This obviously fails in loss networks.

- In Wardrop equilibrium, a standard assumption is that at equilibrium, the cost (delay) between a source and a destination is the same for all users. This does not hold anymore for loss networks.

A particular problem arising in Non-cooperative loss networks has been studied in [3] in the framework of Wardrop equilibrium (with the same topology as the that of the original Braess paradox). However, it is assumed there that the bandwidth required by each user is the same. This assumption may be useful in modeling telephone networks, but is not valid anymore in integrated services. Yet even under the assumption of equal bandwidth requirement, the question of existence and uniqueness of Nash equilibrium and Wardrop equilibrium has not been addressed, and nor have methods for computing the equilibrium been proposed.

In this paper we first present the mathematical models for both Wardrop and Nash equilibria in loss networks, and then show through several examples some properties of their properties. We show that even in the simplest topology of parallel links, neither the 
Wardrop nor the Nash equilibria are unique. Moreover, even the link utilization is not uniquely determined at equilibria. This is in contrast to many other cost criteria in which the Nash equilibrium is known to be unique for the parallel link configuration (see [13]) and in which the link utilizations at Wardrop equilibrium is unique for general topologies (see [14]). We then study a simple routing problem with alternative routes and obtain some paradoxical behavior for the Nash equilibrium. We finally restrict to the special case of parallel links with equal bandwidth requirements of different classes. For that problem we are able to obtain uniqueness properties of the equilibria as well as a convex programming computational approaches to derive them. We provide in that framework a pricing scheme that can be used so as to induce a socially optimal behavior at the Wardrop equilibrium.

The structure of the paper is as follows. We begin in the next section with the model description. Then we present in the consecutive two sections the nonuniqueness and the alternative routing examples. We end up with the section on parallel links with equal bandwidth requirements.

\section{The Model}

Consider a network consisting of $J$ links. Each link $j$ has a capacity of $C_{j}$ bandwidth units. There are $K$ classes of calls, and associated with class- $k$ calls is an arrival rate, $\lambda_{k}$, an average holding time, $\mu_{k}^{-1}$, a bandwidth requirement, $b_{k}$ and a route $r_{k} \subseteq\{1,2, \ldots, J\}$. Denote $\rho_{k}=\lambda_{k} / \mu_{k}$ the class- $k$ offer load. The link capacities and the bandwidth requirements are assumed to be positive integers.

Following Ross [17] (see also Kelly [9]) we have the following. Let $\mathcal{K}$ denote the set of all $K$ classes, i.e. $\mathcal{K}=\{1,2, \ldots, K\}$, and $\mathcal{K}_{j}$ be the set of classes that use link $j$,

$$
\mathcal{K}_{j}=\left\{k \in \mathcal{K}: j \in r_{k}\right\}
$$

Let $m_{k}$ denote the number of class- $k$ calls in the system, and $\mathbf{m}=\left(m_{1}, m_{2}, \ldots, m_{n}\right)$. The state space is

$$
\mathcal{S}=\left\{\mathbf{m}: \sum_{k \in \mathcal{K}_{j}} b_{k} m_{k} \leq C_{j}, \quad j \in\{1,2, \ldots, J\}\right\} .
$$

Next, let $\mathcal{S}_{k}$ be the subset of states for which there is available bandwidth for another arrival of a class- $k$ call, i.e.

$$
\mathcal{S}_{k}=\left\{\mathbf{m} \in \mathcal{S}: \sum_{i \in \mathcal{K}_{j}} b_{i} m_{i} \leq C_{j}-b_{k}, j \in r_{k}\right\} .
$$


Then the steady state distribution for the loss network is

$$
\mathbf{P}\{\mathbf{X}=\mathbf{m}\}=\frac{1}{G} \prod_{k=1}^{K} \frac{\rho_{k}^{m_{k}}}{m_{k} !}, \mathbf{m} \in \mathcal{S},
$$

where

$$
G=\sum_{\mathbf{m} \in \mathcal{S}} \prod_{k=1}^{K} \frac{\rho_{k}^{m_{k}}}{m_{k} !} .
$$

The probability of blocking of a class- $k$ call is

$$
B_{k}=1-\frac{\sum_{\mathbf{m} \in \mathcal{S}_{k}} \prod_{i=1}^{K} \rho_{i}^{m_{i}} / m_{i} !}{\sum_{\mathbf{m} \in \mathcal{S}} \prod_{i=1}^{K} \rho_{i}^{m_{i}} / m_{i} !} .
$$

\subsection{Groups of users}

Assume now that there are $L$ groups which split their demands via the network. It is assumed that group $l$ can use any one of a subset $\mathcal{K}^{l} \subset \mathcal{K}$ of the classes. The set $\mathcal{K}^{l}$ is characterized by a common source and destination to all $k \in \mathcal{K}^{l}$ as well as a common parameters $b_{l}$ and $\mu_{l}$, and a total demand of rate $\lambda_{l}$. Group $l$ sends the fraction $p_{l, i}$ of the its demand via the route $r_{i}$. Then $\lambda_{l, i}=\lambda_{l} p_{l, i}\left(i \in \mathcal{K}^{l}\right)$ is the rate of Poisson stream that user $l$ ships via route $r_{i}$, and $\rho_{l, i}=\lambda_{l, i} / \mu_{l}$. Define $\mathbf{r}_{l}=\left(\rho_{l, 1}, \ldots, \rho_{l, n_{l}}\right)$.

We shall now consider two equilibria concepts depending on whether to each single group there is associated a single decision maker, or whether each group only defines a large set of users having some common parameters.

\subsection{Finite number of users: Nash equilibrium}

Here, group $l$ has a single decision maker and is just identified with a single "user" (in the terminology of [13]). User $l$, we can view the set $\mathbf{r}_{l}=\left(\rho_{l, 1}, \ldots, \rho_{l, n_{l}}\right)$ as routing decision variables (instead of the splitting probabilities $p_{l, i}, i \in \mathcal{K}^{l}$ ).

The goal of user $l$ is to minimize its average blocking probability. We can thus define the following cost (which is proportional to the average blocking probability of user $l$ ):

$$
J_{l}\left(\mathbf{r}_{1}, \mathbf{r}_{2}, \ldots, \mathbf{r}_{l}, \ldots, \mathbf{r}_{L}\right)=\sum_{n \in \mathcal{K}^{l}} p_{l, n} B_{n} .
$$

Definition 1 A vector $\tilde{\mathbf{r}}_{l}, l=1,2, \ldots, L$ is called a Nash equilibrium if for each user $l, \tilde{\mathbf{r}}_{l}$ minimizes the cost function (7) given that other users' decisions are $\tilde{\mathbf{r}}_{m}, m \neq l$. In other words,

$$
J_{l}\left(\tilde{\mathbf{r}}_{1}, \tilde{\mathbf{r}}_{2}, \ldots, \tilde{\mathbf{r}}_{L}\right)=\min _{\mathbf{r}_{l} \in \mathbf{R}_{l}} J_{l}\left(\tilde{\mathbf{r}}_{1}, \tilde{\mathbf{r}}_{2}, \ldots, \mathbf{r}_{l}, \ldots, \tilde{\mathbf{r}}_{L}\right), \quad l=1,2, \ldots, L
$$


where $\mathbf{R}_{l}$ is the set of possible values of vector $\mathbf{r}_{l}=\left(\rho_{l, 1}, \rho_{l, 2}, \ldots, \rho_{l, K}\right)$ with nonnegative components, such that the total rate to be shipped is $\lambda_{l}$. The last constraint is written as:

$$
\sum_{n=1}^{K} \rho_{l, n} \mu_{l}=\lambda_{l}
$$

\subsection{Atomless users: Wardrop equilibrium}

In case of a very large number of users, a standard way of modeling the equilibrium concept considers a user to be atomless, i.e. the decisions of a single user have a negligible influence on performance measures of other users. This is amounts to considering a continuum of players, each of which has mass zero. This modeling gives rise to the Wardrop equilibrium [18] that turns out to be much easier to handle than Nash equilibrium. In particular, it can often be computed by solving some convex minimization problem (which can be interpreted as an equivalent problem with a single user and some other cost function. We shall come back to this aspect later), see [14].

Definition 2 A vector $\tilde{\mathbf{r}}_{l}, l=1,2, \ldots, L$ is called a Wardrop equilibrium if for each group $l$ the following holds. For each group $l$, let $\mathcal{K}_{*}^{l} \subset \mathcal{K}$ be the set of routes actually used, that is the set of classes such that $\rho_{l, k}>0, k \in \mathcal{K}^{l}$. Then

$$
B_{k}(\tilde{\mathbf{r}}) \leq B_{m}(\tilde{\mathbf{r}})
$$

for each $k \in \mathcal{K}_{*}^{l}$ and each $m \in \mathcal{K}^{l}$.

The definition is a formulation of the two Wardrop principles: all used passes of a given class have the same cost, and this cost is not greater than the cost of unused paths. In our case, the cost is identified with blocking probabilities. Note that the original Wardrop equilibrium was restricted to the case that a class can be identified with a pair of source destination, and it did not include our setting in which different classes can have different blocking probabilities between the same source and destination along the same paths, since they require different amount of bandwidth. Moreover, the cost of a path is usually referred to as the sum of link costs along that path, which does not apply to our setting. However, extensions to multiclass situations which cover our model do exist, see e.g. [14, Chap. 3]. Yet, as we shall see, the conditions for the uniqueness of equilibria presented there do not hold in our case.

Theorem 1 There exists a Wardrop equilibrium.

INRIA 
Proof. We note that the set of policies is bounded. The proof then follows directly from Theorem 3.1 in [14], which states that a solution exists to a set of some variational inequalities. Then, Theorem 3.14 in [14] establishes that the solution to these inequalities is indeed a Wardrop equilibrium.

\section{Non-uniqueness of equilibrium}

\subsection{Wardrop Equilibrium}

We now consider the following example. There are two parallel links. The first one, denoted

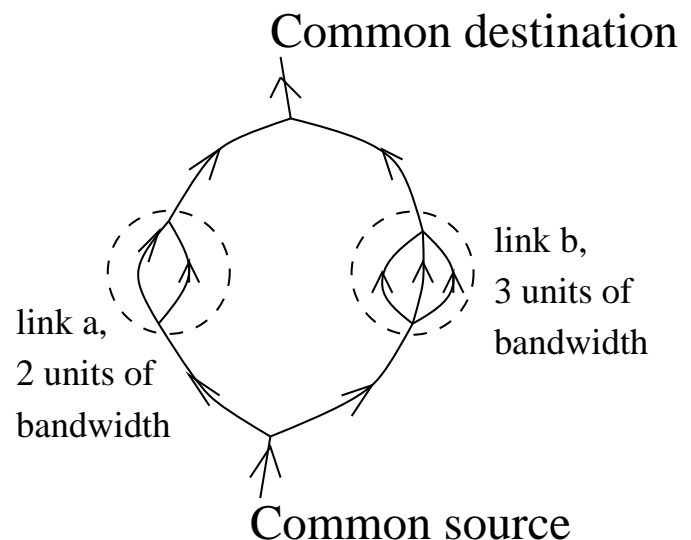

Figure 1: Routing to parallel links

by $a$, consists of 2 bandwidth units, and the second one, denoted by $b$, consists of 3 bandwidth units. There are 2 groups that wish to ship traffic from a common source to a common destination. Each arrival of Group I requires 1 bandwidth units, whereas an arrival of Group II requires only 2 bandwidth unit.

We assume that all users can send traffic through both links. We denote the classes by the tuple $(l, j)$ where $l=I, I I$ stands for the user and $j=a, b$ stands for the link. Thus $\mathcal{K}=\{(I, a),(I, b),(I I, a),(I I, b)\}$. All classes have the same source and destination. We have $\mathcal{K}^{I}=\{(I, a),(I, b)\}$ and $\mathcal{K}^{I I}=\{(I I, a),(I I, b)\}$. We have in particular the bandwidth requirements: $b_{(I, a)}=b_{(I, b)}=1$ and $b_{(I I, a)}=b_{(I I, b)}=2$.

Note: this network is not equivalent to a single parallel link with capacity 5 , since we do not allow a single call to be split between link $a$ and link $b$.

$\mathrm{RR} \mathrm{n}^{\circ} 4405$ 
The state space is the product of the state space of each separate link. A given state of link $j$ is a tuple $(x, y)$ where $x$ is the number of ongoing calls of type $\mathrm{I}$, and $y$ is the number of ongoing calls of type II over link $j$. The state space $S_{j}$ of link $j$ is thus,

$$
S_{a}=\{(0,0),(1,0),(2,0),(0,1)\}
$$

and

$$
S_{b}=\{(0,0),(1,0),(2,0),(3,0),(0,1),(1,1)\} .
$$

For user $I I$, a call is blocked on link $a$ if it is not empty. This occurs with probability

$B_{(I I, a)}=1-\frac{\rho_{(I I, a)}^{0} \rho_{(I, a)}^{0}}{\rho_{(I I, a)}^{0} \rho_{(I, a)}^{0}+\rho_{(I I, a)}^{0} \rho_{(I, a)}^{1}+\rho_{(I I, a)}^{1} \rho_{(I, a)}^{0}+\rho_{(I I, a)}^{0} \rho_{(I, a)}^{2} / 2 !}=1-\frac{1}{1+\rho_{(I, a)}^{1}+\rho_{(I I, a)}^{1}+\rho_{(I, a)}^{2} / 2}$

For group $I I$, a call is not blocked on link $b$ if there are no ongoing calls of that group over that link and there is at most one call of class I over link $b$. Thus

$$
B_{(I I, b)}=1-\frac{1+\rho_{(I, b)}}{1+\rho_{(I, b)}+\rho_{(I, b)}^{2} / 2+\rho_{(I, b)}^{3} / 6+\rho_{(I I, b)}+\rho_{(I I, b)} \rho_{(I, b)}} .
$$

For group I, link $a$ is blocked if there is either one call of user II on that link or there are 2 calls of group I. This occurs with probability

$$
B_{(I, a)}=\frac{\rho_{(I I, a)}+\rho_{(I, a)}^{2} / 2}{1+\rho_{(I, a)}^{1}+\rho_{(I I, a)}^{1}+\rho_{(I, a)}^{2} / 2}
$$

For group I, link $b$ is blocked if there is either one call of user II and one of user I on that link or there are 3 calls of group I. This occurs with probability

$$
B_{(I, b)}=\frac{\rho_{(I I, b)} \rho_{(I, b)}+\rho_{(I, b)}^{3} / 6}{1+\rho_{(I, b)}+\rho_{(I, b)}^{2} / 2+\rho_{(I, b)}^{3} / 6+\rho_{(I I, b)}+\rho_{(I I, b)} \rho_{(I, b)}} .
$$

We considered the following parameters: $\mu_{k}=1, \forall k \in \mathcal{K}$ (this choice will allow us to interchange $\lambda_{k}$ with $\rho_{k}$ ) $\lambda_{I}=1, \lambda_{I I}=0.2$. (Recall that $\mu_{k}^{-1}$ is the average call duration of class $k$, and that $\rho_{k}=\lambda_{k} / \mu_{k}$.)

We now solved the set of four equations

$$
\begin{aligned}
B_{(l, a)}(\mathbf{r}) & =B_{(l, b)}(\mathbf{r}), \quad l=I, I I \\
\rho_{(l, a)}+\rho_{(l, b)} & =\lambda_{l}, \quad l=I, I I .
\end{aligned}
$$

which gave us two sets of solutions. Yet only one of these solutions satisfied the nonnegativity constraints $\rho_{(l, j)} \geq 0, \forall l, j$. This solution is given by

$$
\rho_{(I, a)}=0.2386, \rho_{(I, b)}=0.7614, \rho_{(I I, a)}=0.0696, \rho_{(I I, b)}=0.1304 .
$$


The obtained rejection probabilities are

$$
B_{(I, a)}=B_{(I, b)}=0.07355, \quad B_{(I I, a)}=B_{(I I, b)}=0.2522 .
$$

The fact that equation (10)-(11) have a single solution does not mean that there is a single Wardrop equilibrium. We should still check for Wardrop equilibria in which for some $l$ and $j, \rho_{(l, j)}=0$. Checking for the different possibilities, we came out with the following second Wardrop equilibrium:

$$
\rho_{(I, a)}=0.4567, \rho_{(I, b)}=0.5433, \rho_{(I I, a)}=0, \rho_{(I I, b)}=0.2 .
$$

The obtained rejection probabilities are

$$
B_{(I, a)}=B_{(I, b)}=0.06681, \quad B_{(I I, a)}=0.3594, \quad B_{(I I, b)}=0.2384 .
$$

We see that here we have $B_{(I, a)}=B_{(I, b)}$ but $B_{(I I, a)} \neq B_{(I I, b)}$. But this agrees with the Wardrop principle that says that the cost of a path that is used (in our case route $b$ that is used by user II), should be no larger than that of any path not used (in our case, route $a$, which has indeed larger blocking probability).

We conclude that we have here indeed two distinct Wardrop equilibria. In the "standard" Wardrop equilibrium setting, in which the cost of a class depends only on the source and destination, we know that Wardrop equilibrium need not be unique either, but that the link utilization are uniquely defined. In other words, we have there unique values of $\rho_{j}=\sum_{l} \rho_{l, j}$ for every link $j$ at equilibrium, see e.g. [14, p. 43] or even some multi-class extensions, see [1]. In our case not only the equilibria are not unique, but we see that also the link utilization at equilibria differ.

\subsection{Nash Equilibrium}

Next, we consider the same example in the context of Nash equilibrium with two players, I and II corresponding to the two groups we described in the previous section. Consider the following parameters: $\lambda_{I}=\lambda_{I I}=0.9, \mu_{I}=1, \mu_{I I}=2$.

Then three equilibria are obtained, The first is

$$
\lambda_{(I, a)}=0.04435, \lambda_{(I I, a)}=0.5335, \lambda_{(I, b)}=0.85565, \lambda_{(I I, b)}=0.3665,
$$

the second

$$
\lambda_{(I, a)}=0.2549, \lambda_{(I I, a)}=0.3955, \lambda_{(I, b)}=0.6451, \lambda_{(I I, b)}=0.5045,
$$

and the third

$$
\lambda_{(I, a)}=0, \lambda_{(I I, a)}=0.56055, \lambda_{(I, b)}=0.9, \lambda_{(I I, b)}=0.33945 .
$$




\section{Alternative routing example}

Consider the loss networks in Figures 2 and 3.

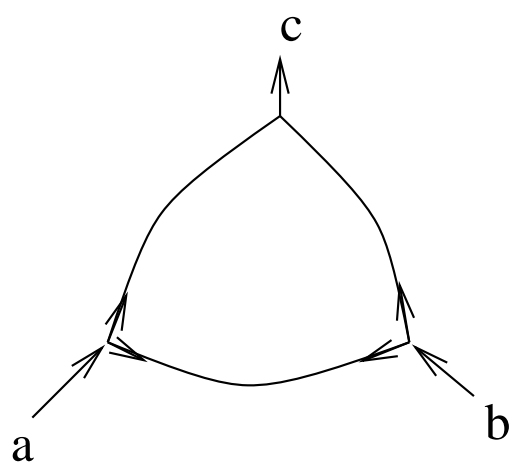

Figure 2: Network A with one forwarding bidirectional link

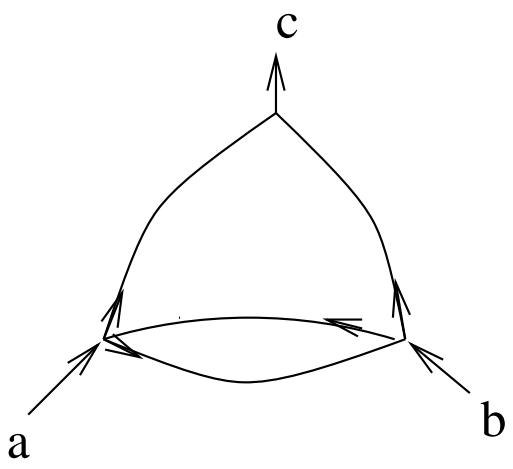

Figure 3: Network B with two forwarding unidirectional links

These types of networks have been studied in $[7,8]$ in the context of delay minimization where the delays of links correspond to $\mathrm{M} / \mathrm{M} / 1$ queues with infinite buffers. Here we study some of their properties when the objectives to be minimized are blocking probabilities.

There are two source nodes, $a$ and $b$, and one common destination $c$. A call arriving at a source node can choose either a direct path of one hop, or an alternative path that requires two hops. The paths are thus $a c$ and $a b c$ for calls arriving at $a$, and $b c$ and $b c a$ for calls arriving at $b$.

We consider finite link capacities $C_{a b}, C_{a c}$; furthermore, in network A there is one common bidirectional link with capacity $C_{b c}$, whereas in network B there are instead two unidirectional links with capacities $C_{a b}$ and $C_{b a}$, respectively.

To make the problem nontrivial we make appropriate assumptions that guarantee that the forwarding links have an influence on the equilibrium, i.e. they may be responsible for blocking.

For network A we shall assume that $C_{a b}<C_{a c}+C_{b c}$. Indeed, if this condition is not satisfied then we can replace the whole system with a single source ( $a$ and $b$ combined) and a single link between that source and the destination $c$ with capacity $C_{a c}+C_{b c}$, without affecting the blocking probabilities (under any policy).

For similar reasons, we assume for network B that $C_{a b} \leq C_{b c}$ and $C_{b a}<C_{a c}$.

Denote the total demands by $\lambda_{a}$ (and $\lambda_{b}$ ) which corresponds to the total rate to be shipped to $c$ from source $a$ (and from source $b$, respectively). 


\subsection{Wardrop equilibrium}

We shall establish the following main structural result.

Proposition 1 Consider network $A$ assuming that $C_{a b}<C_{a c}+C_{b c}$ or network $B$ under the condition that $C_{a b} \leq C_{b c}$ and $C_{b a}<C_{a c}$. Then for both networks, there is no mutual forwarding at Wardrop equilibrium. In other words, if a strictly positive fraction of calls arriving to $a$ is routed through $b$, then no jobs arriving at $b$ are routed through $a$, and vice versa.

Proof. Consider network A. Assume that at some Wardrop equilibrium a strictly positive fraction of calls arriving to $a$ is routed through $b$ and vice versa.

Direct connections from $b$ to $c$ are only blocked if link $b c$ is full, whereas indirect connections from $a$ to $c$ that use $b$ can be blocked not only if $b c$ is full but also if $a b$ is full. The latter may occur even when link $b c$ is not full. The blocking at $a b$ of such indirect calls has positive probability since there is mutual forwarding and since $C_{a b}<C_{a c}+C_{b c}$. This implies that $B_{b c}<B_{a b c}$.

Since $a b c$ has positive flow of connections from $a$, it follows from Wardrop principle that $B_{a b c} \leq B_{a c}$. We thus conclude that $B_{b c}<B_{a c}$. By a symmetric argument we can now show the opposite, i.e. $B_{b c}>B_{a c}$, and obtain a contradiction. We thus conclude that there cannot be mutual forwarding.

A similar argument holds for network B: it follows again that that $B_{b c}<B_{a b c}$. Assuming mutual forwarding, due to Wardrop principle, since $a b c$ has positive flow of connections from $a$, it follows that $B_{a b c} \leq B_{a c}$. We conclude that $B_{b c}<B_{a c}$. A symmetric argument leads to the opposite relation under mutual forwarding which is impossible.

Corollary 1 Consider the symmetric case $C_{a c}=C_{b c}$. Consider either network $A$ assuming that $C_{a b}<2 C_{a c}$, or network $B$ assuming that $C_{a b}=C_{b a}<C_{a c}$. At Wardrop equilibrium only direct links are used.

Proof. Consider either network A or B. Assume that at equilibrium, a strictly positive amount of flow from $a$ goes through $b$. Then due to the previous Proposition, all flow from $b$ goes through $b c$. The total flow over $b c$ is strictly larger than through $a c$. Hence we have $B_{a c}<B_{b c} \leq B_{a b c}$. Thus Wardrop principles are violated, since the used path $a b c$ has strictly larger cost than another path between the same origin and destination. 


\subsection{Nash equilibrium}

In this subsection we consider a particular example of Network A in which each link has 1 unit capacity. We assume symmetric demands $\bar{\lambda}=\lambda_{a}=\lambda_{b}$.

Denote for simplicity $x_{i}$ the amount of flow on the direct link from source $i=a, b$ to the destination, and let $y_{i}$ be the amount of flow on the indirect link from source $i=a, b$.

We then have

$$
B_{a c}=\frac{x_{a}\left(1+x_{b}\right)+y_{b}\left(1+x_{b}\right)}{1+x_{a}+x_{b}+y_{a}+y_{b}+x_{a} y_{a}+x_{b} y_{b}+x_{a} x_{b}}=\frac{x_{a}\left(1+x_{b}\right)+\left(\bar{\lambda}-x_{b}\right)\left(1+x_{b}\right)}{1+2 \bar{\lambda}+x_{a}\left(\bar{\lambda}-x_{a}\right)+x_{b}\left(\bar{\lambda}-x_{b}\right)+x_{a} x_{b}}
$$

and

$$
B_{a b c}=1-\frac{1+x_{a}}{1+2 \bar{\lambda}+x_{a}\left(\bar{\lambda}-x_{a}\right)+x_{b}\left(\bar{\lambda}-x_{b}\right)+x_{a} x_{b}}
$$

The cost for group $a$ is given by

$$
\begin{aligned}
J_{a} & =x_{a} B_{a c}+\left(\bar{\lambda}-x_{a}\right) B_{a b c} \\
J_{b} & =x_{b} B_{b c}+\left(\bar{\lambda}-x_{b}\right) B_{b a c}
\end{aligned}
$$

We identify a symmetric Nash equilibrium as follows. For a given $x_{b}$, we find the best response $x_{a}^{*}\left(x_{b}\right)$ for a fixed $x_{b}$ by differentiating $J_{a}$ with respect to $x_{a}$ and equating to zero, i.e

$$
x_{a}^{*}\left(x_{b}\right)=\arg \min _{x_{a}} J_{a}
$$

We then solve the equation

$$
x_{b}=x_{a}^{*}\left(x_{b}\right) .
$$

If the solution is within $[0, \bar{\lambda}]$ then it is a Nash equilibrium.

We now considered the following parameters: $\bar{\lambda}=\lambda_{a}=\lambda_{b}=0.3$.

We now solved the equation (12)-(13). Under the exact analysis, the Nash equilibrium is given by

$$
x_{a}^{*}=0.1932, x_{b}^{*}=0.1932, \quad J_{a}^{*}=J_{b}^{*}=0.0721 .
$$

Now, we consider the scenario where the common bidirectional link is removed, the trivial Nash equilibrium in this case is

$$
x_{a}^{*}=0.3, \quad x_{b}^{*}=0.3, \quad J_{a}^{*}=J_{b}^{*}=0.0692 .
$$

We observe that the cost function of both players increase when adding the link and the best performance can be achieved in the absence of the bidirectional link.

INRIA 
The above equilibrium is the unique one we obtained for the problem. In particular, sending flows only through direct routes is not an equilibrium. Indeed, if one player sends all its flow through a direct route, the best response for the other player is not to send its flow on its own direct route, but instead, to split its flow as follows: 0.2125 on the direct route and 0.0875 on the indirect one.

The simple problem we introduced here is an example of a Braess-type paradox (which does not occur in the Wardrop equilibrium, in which the indirect links are not used). This paradox has previously been obtained for expected delays as performance measures within the same network topology, see $[7,8]$.

\section{Parallel links with equal bandwidth requirements}

We now consider the special case of bandwidth requirement of one bandwidth unit by each connection. In that case, the blocking probabilities becomes the same for all users that have the same source and destination. Still, the blocking probabilities along one path remains a complex function of the flows of all classes that share at least one common link along that path.

This problem disappears, however, in the special case of the parallel links topology, which had been extensively studied (see e.g. [13]), see e.g. the topology of the example in Section 3. In that case a link can be identified with a whole path and with a whole traffic class. We shall furthermore assume that the duration of a call has the same exponential distribution for all users, with parameter 1.

Assume that link $i$ has capacity $C_{i}$. Then the blocking probability over that link is given by the Erlang loss formula, which is only a function of the total link flow $\lambda(i)$ :

$$
B_{i}(\lambda(i))=\frac{\lambda(i)^{C_{i}} / C !}{\sum_{j=1}^{C_{i}} \lambda(i)^{j} / j !}
$$

(this is the Erlang loss formula, also known as the Erlang B formula). Note that this is a monotone increasing function of $\lambda(i)$.

\subsection{Wardrop equilibrium}

Next we present a computation approach for the Wardrop equilibrium. Denote the total demand by $\Lambda=\sum_{l=1}^{L} \lambda_{l}$. The link utilization at equilibrium can be obtained using a standard transformation into the following equivalent global optimization problem [14, p.

$\mathrm{RR} \mathrm{n}^{\circ} 4405$ 
35 ] in which the cost to be minimized is

$$
T(\lambda):=\sum_{i \in \mathcal{K}} \int_{0}^{\lambda(i)} B_{i}(z) d z
$$

where $\lambda=(\lambda(i), i \in \mathcal{K})$. The optimization problem is given by

$$
\min T(\lambda) \quad \text { s.t. } \sum_{i \in \mathcal{K}} \lambda(i)=\Lambda, \lambda(i) \geq 0, \forall i=1, \ldots, \mathcal{K} .
$$

We now show that the cost function has a simple explicit form.

Theorem 2 The unique link utilization at Wardrop equilibrium for our parallel link problem is given by solving (15) where

$$
T(\lambda)=-\sum_{i \in \mathcal{K}} \log G_{C_{i}}(\lambda(i)), \quad \text { where } \quad G_{N}(x)=\sum_{i=0}^{N} x^{i} / i !
$$

Proof. We can write the blocking probability of link $i$ as

$B(\lambda(i))=1-\frac{\sum_{j=1}^{C_{i}-1} \lambda(i)^{j} / j !}{\sum_{j=1}^{C_{i}} \lambda(i)^{j} / j !}=1-\frac{\mathrm{d}\left(\sum_{j=1}^{C_{i}} \lambda(i)^{j} / j !\right) / \mathrm{d} \lambda(i)}{\sum_{j=1}^{C_{i}} \lambda(i)^{j} / j !}=1-\frac{\mathrm{d} \log \left(\sum_{j=1}^{C_{i}} \lambda(i)^{j} / j !\right)}{\mathrm{d} \lambda(i) .}$

Taking the integral in (14) we obtain (16) (up to the additive constant $\Lambda$ that may be omitted, which does not influence the solution of (15)).

The Wardrop equilibria has another interesting interpretation. Assume that each link belongs to another provider, and that we wish to dispatch a demand of $\Lambda$ between them. Assume that the utility for provider $i$ of dispatching an amount $\lambda(i)$ to it is $G_{C_{i}}(\lambda(i))$. Then the optimization problem (15) coincides with the one that is frequently used for obtaining the well known proportional fairness assignment, see e.g. [11]. Thus in our interpretation, the Wardrop equilibrium is proportional fair.

\subsection{Pricing for minimizing rejections}

We consider here a two phase problem. The network manager determines the configuration of flows that minimizes the expected rejection probabilities. Then she assigns prices for links. At a second stage, we consider the Wardrop equilibrium that is obtained when users take decisions so as to minimize the link costs. We determine the prices that imply that the resulting Wardrop equilibrium achieves the initial objective of the network manager.

INRIA 
The global cost that the network manager wishes to minimize is $J=\sum_{i} J^{i}(\lambda)$ where

$$
J^{i}(\lambda)=p_{i} B_{i}(\lambda(i))=\frac{\lambda(i)}{\sum_{j} \lambda(j)} B_{i}(\lambda(i)) .
$$

This problem has a unique solution due to the following Lemma whose proof is a special case of Lemma 2 that is proved in the Appendix.

Lemma $1 J^{i}$ is convex in its argument.

Proposition 2 Set the following link costs:

$$
f_{i}(\lambda(i))=\left(C_{i}+1\right) B_{i}\left(\lambda_{i}\right)+\lambda(i)\left(B_{i}\left(\lambda_{i}\right)^{2}-B_{i}\left(\lambda_{i}\right)\right) .
$$

Then the corresponding Wardrop equilibrium minimizes the average rejection probability $J(\lambda)$.

Proof. The Wardrop equilibrium for the routing problem with link costs $f_{i}$ is the solution of the equivalent global optimization problem [14, p. 35] in which the cost to be minimized is

$$
T(\lambda):=\sum_{i \in \mathcal{K}} \int_{0}^{\lambda(i)} f_{i}(z) d z
$$

where $\lambda=(\lambda(i), i \in \mathcal{K})$. The optimization problem is given by

$$
\min T(\lambda) \quad \text { s.t. } \sum_{i \in \mathcal{K}} \lambda(i)=\Lambda, \lambda(i) \geq 0, \forall i=1, \ldots, \mathcal{K} .
$$

Hence it suffices to show that $\mathrm{d} J^{i}(x) / d x=f_{i}(x) / \sum_{j} \lambda(j)$.

We first note that

$$
\frac{\mathrm{d} B_{i}(x)}{\mathrm{d} x}=\frac{x^{N-1} /(N-1) !}{G_{N}(x)}-\frac{G_{N-1}(x) x^{N} / N !}{G_{N}(x)^{2}} .
$$

Hence

$$
\begin{aligned}
\frac{\mathrm{d} x B_{i}(x)}{\mathrm{d} x} & =B_{i}(x)+x B_{i}^{\prime}(x)=B_{i}(x)+\frac{N x^{N} / N}{G_{N}(x)}-x \frac{x^{N}}{N !} \frac{G_{N}-x^{N} / N !}{G_{N}(x)^{2}} \\
& =(N+1) B_{i}(x)+x\left(B_{i}(x)^{2}-B(x)\right)=f_{i}(x) .
\end{aligned}
$$

This establishes the proof.

$\mathrm{RR} \mathrm{n}^{\circ} 4405$ 


\subsection{Nash equilibrium}

In [13], the following assumptions are made on the cost functions $J_{l}$.

- G. $J_{l}$ is the sum of link cost functions $J_{l}^{i}$ for all links $i \in \mathcal{I}$; it is a continuous function and convex in $\lambda_{l, i}$. Furthermore, $J_{l}^{i}$ is continuously differentiable with respect to $\lambda_{l, i}$. Denote this partial derivative by $K_{l}^{i}=\frac{\partial J_{l}^{i}}{\partial \lambda_{l, i}}$.

- A1. $J_{l}^{i}$ is a function of two arguments: user l's flow on link $i$ and the total flow on that link. In other words, $J_{l}^{i}(\lambda)=\bar{J}_{l}^{i}\left(\lambda_{l, i}, \lambda(i)\right)$.

- A2. $\bar{J}_{l}^{i}$ is increasing in both arguments.

- A3. $K_{l}^{i}=K_{l}^{i}\left(\lambda_{l, i}, \lambda(i)\right)$ is now a function of two arguments. We assume that it is strictly increasing in both.

We first note that under our assumptions, the cost functions satisfy assumptions G, which guarantees the existence of the Nash [13]. In particular, we show the following in the Appendix:

Lemma $2 J_{l}^{i}$ is indeed convex in $\lambda_{i, l}$

Secondly, we note that the costs satisfy assumption A1-A3, which guarantees that it is unique. $^{1}$

The following Kuhn-Tucker conditions are sufficient and necessary for some $\lambda^{*}$ to be a Nash equilibrium [13]. There exist some non-negative Lagrange multipliers $\alpha^{l}, l=1, \ldots, L$ such that for all $l$ and $i$,

$$
K_{l}^{i}\left(\lambda^{*}\right) \geq \alpha^{l},
$$

and whenever $\lambda_{l, i}^{*}>0$, we have

$$
K_{l}^{i}\left(\lambda^{*}\right)=\alpha^{l}
$$

In addition,

$$
\sum_{i \in \mathcal{K}} \lambda_{l, i}=\lambda_{l}
$$

Assume that some assignment $\lambda^{*}=\left(\lambda_{l, i}^{*}\right)$ is a Nash equilibrium with the following property: all users send positive flow through all links. We show that for this special case there is too, a simple method of computing the link utilization at equilibrium. (Note that

\footnotetext{
${ }^{1}$ We note that the cost does not satisfy other sets of assumptions in [13], such as assumption B or assumptions $\mathrm{C}$
}

INRIA 
at Nash equilibrium it is impossible that through some link the total flow would be zero.) We note that our assumption implies that (19) holds for all $i$ and $l$. We note that

$$
K_{l}^{i}(\lambda)=\frac{\lambda_{i, l}}{\lambda_{l}} \frac{\mathrm{d} B_{i}(\lambda(i))}{\mathrm{d} \lambda(i)}+\frac{1}{\lambda_{l}} B_{i}(\lambda(i))
$$

Let $\beta:=\sum_{l=1}^{L} \alpha^{l} / \lambda_{l}$. Thus, summing over all $l$, we get for each $i$

$$
\lambda(i) \frac{\mathrm{d} B_{i}\left(\lambda^{*}(i)\right)}{\mathrm{d} \lambda^{*}(i)}+L B_{i}\left(\lambda^{*}(i)\right)=\beta .
$$

Taking the sum also in (20) yields

$$
\sum_{i \in \mathcal{K}} \lambda^{*}(i)=\Lambda
$$

But these two relations are precisely the Kuhn-Tucker conditions for an optimization problem faced by a single user whose cost function is given by

$$
T(\lambda)=\sum_{i \in \mathcal{K}} \lambda(i) B_{i}(\lambda(i))+(L-1) \int_{0}^{\lambda(i)} B_{i}(z) d z .
$$

We can now compute the last integral as we did in the Wardrop equilibrium and obtain:

Theorem 3 Consider the parallel link problem. Then there exists a unique Nash equilibrium. If it has the property that all users send positive flow through all links then the link utilization at the Nash equilibrium is given by solving (15) where

$$
T(\lambda)=\sum_{i \in \mathcal{K}}\left(\frac{\lambda(i) B_{i}(\lambda(i))}{L-1}-\log G_{C_{i}}(\lambda(i))\right) .
$$

(note that we eliminated a constant term from the integral, which does not influence the solution of the optimization problem, and also divided the whole cost by the constant $L-1$ ).

Remark 1 We know from [6] that Nash equilibria converges to the Wardrop equilibrium as the number of players grow to infinity. This seems to be confirmed by the fact that the objective function (21) corresponding to the Nash equilibrium which we compute using the optimization problem (15) converges to the objective function (16) that is used for computing the Wardrop equilibrium.

$\mathrm{RR} \mathrm{n}^{\circ} 4405$ 


\section{Appendix: proof of Lemma 2}

We recall that for a $N \geq 0$

$$
B_{k, N}=\frac{\rho_{k}^{N} / N !}{G_{N}\left(\rho_{k}\right)}
$$

where $G_{N}(\rho)=\sum_{i=0}^{N} \rho^{i} / i$ !. It follows from representation (22) that it is sufficient to prove that the function

$$
f_{N}\left(\rho_{l}\right)=\rho_{l} B_{k, N}(\rho)
$$

where $\rho=\left(\rho_{1}, \rho_{2}, . ., \rho_{L}\right)$ and $\rho=\sum_{l=1}^{L} \rho_{l}$, is convex in $\rho_{l}$. We have

$$
\frac{\partial f_{N}\left(\rho_{l}\right)}{\partial \rho_{l}}=B_{k, N}(\rho)+\rho_{l} B_{k, N}^{\prime}(\rho)
$$

and the second derivative of function $f_{N}$ is

$$
\frac{\partial^{2} f_{N}\left(\rho_{\mathbf{l}}\right)}{\partial \rho_{l}^{2}}=2 B_{k, N}^{\prime}(\rho)+\rho_{l} B_{k, N}^{\prime \prime}(\rho)
$$

Now, we must prove that the second derivative of the function $f_{N}\left(\rho_{l}\right)$ is positive. We begin by taking the derivative of $B_{k, N}$. After some simplification, we obtain

$$
B_{k, N}^{\prime}(\rho)=\frac{1}{G_{N}^{2}(\rho)} \frac{\rho^{N-1}}{N !} \sum_{i=0}^{N} \frac{\rho^{i}}{i !}(N-i)
$$

which is positive. Hence from (25), if $B_{k, N}^{\prime \prime}(\rho)$ is nonnegative, then the function $f_{N}$ is convex in $\rho_{l}$. Now if $B^{\prime \prime}(\rho)$ is negative then we have

$$
\frac{\partial^{2} f_{N}\left(\rho_{\mathbf{l}}\right)}{\partial \rho_{l}^{2}}=2 B_{k, N}^{\prime}(\rho)+\rho_{l} B_{k, N}^{\prime \prime}(\rho) \geq 2 B_{k, N}^{\prime}(\rho)+\rho B_{k, N}^{\prime \prime}(\rho)
$$

The last term is exactly the second derivative of function $g_{N}$ where

$$
g_{N}(\rho)=\rho B_{k, N}(\rho) .
$$

It is positive since $g_{N}$ is convex in $\rho$, see [12]. This establishes the proof.

\section{Acknowledgements}

This paper was carried out while Vyacheslav Abramov was with INRIA Sophia Antipolis in summer 2000. Their hospitality is very much appreciated. We wish to thank Laura Wynter for useful discussions and suggestions.

INRIA 


\section{References}

[1] E. Altman and H. Kameda, "Equilibria for Multiclass Routing in Multi-Agent Networks", 40th IEEE Conference on Decision and Control, Orlando, Florida, U.S.A., Dec. 2001.

[2] T. Basar and G. Olsder, Dynamic Noncooperative Game Theory, Academic Press, London, 1982.

[3] Bean, N.G., Kelly, F.P. and Taylor, P.G. (1997) Braess's paradox in a loss network. $J$. Appl. Prob., 34, 155-159.

[4] Braess, D. (1968) Uber ein Paradoxen der Verkehrsplannung. Unternehmens-forschung, 12, 258-268.

[5] P. Gupta and P. R. Kumar. "A system and traffic dependent adaptive routing algorithm for Ad Hoc networks", In Proceedings of the 36th IEEE Conference on Decision and Control, pages 2375-2380, San Diego, USA, Dec. 1997.

[6] A. Haurie and P. Marcotte, "On the relationship between Nash-Cournot and Wardrop Equilibria", Networks, 15, pp. 295-308, 1985.

[7] H. Kameda, E. Altman, T. Kozawa and Y. Hosokawa, "Braess-like paradoxes of Nash Equilibria for load balancing in distributed computer systems", IEEE Trans. on Automatic Control, 45, No. 9, 1687-1691, 2000.

[8] H. Kameda, H. Yoshihisa and O. Pourtallier, "Effects of symmetry on Braess-like paradoxes in distributed computer systems - a numerical study", Proceedings of the 40th IEEE Conference on Decision and Control, Orlando, Florida, Dec. 4-7, 2001.

[9] F.P. Kelly (1991) "Loss networks", Ann. Appl. Prob., 1, 319-378.

[10] Y.A. Korilis, A. A. LAZAR and A. Orda, Avoiding the Braess paradox in noncooperative networks. J. Appl. Prob., 36, 211-222, 2999

[11] A. Maulloo F.P. Kelly and D. Tan. Rate control in communication networks: shadow prices, proportional fairness and stability. J. Oper. Res. Society, 49:237-252, 1998.

[12] K. R. Krishnan, The convexity of loss rate in an Erlang loss system and sojourn in an Erlang delay system with respect to arrival and service rates, IEEE Transactions on Communications, vol. 38, No. 9, September 1990.

$\mathrm{RR} \mathrm{n}^{\circ} 4405$ 
[13] Orda, A., Rom, R. and Shimkin, N. (1993) Competitive routing in multi-user communication networks. IEEE/ACM Trans. Network. 1, 510-521.

[14] M. Patriksson The Traffic Assignment Problem: Models and Methods VSP BV, P.O. Box 346, 3700 AH Zeist, The Netherlands, 1994.

[15] A. C. Pigou. The Economics of Welfare. McMillan \& Co., London, 1920.

[16] J.B. Rosen (1965) Existance and uniqueness of equilibrium points for concave $N$-person games. Econometrica, 33, 520-534.

[17] K.W. Ross (1995) Multiservice Loss Models for Broadband Telecommunication Networks. Springer-Verlag, Berlin.

[18] Wardrop, J.G. (1952) Some theoretical aspects of road traffic research. Proc. Inst. Civil Eng., Part 2, 1, 325-378. 


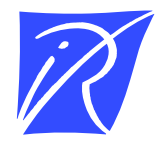

Unité de recherche INRIA Sophia Antipolis

2004, route des Lucioles - BP 93 - 06902 Sophia Antipolis Cedex (France)

Unité de recherche INRIA Lorraine : LORIA, Technopôle de Nancy-Brabois - Campus scientifique 615, rue du Jardin Botanique - BP 101 - 54602 Villers-lès-Nancy Cedex (France)

Unité de recherche INRIA Rennes : IRISA, Campus universitaire de Beaulieu - 35042 Rennes Cedex (France)

Unité de recherche INRIA Rhône-Alpes : 655, avenue de l'Europe - 38330 Montbonnot-St-Martin (France)

Unité de recherche INRIA Rocquencourt : Domaine de Voluceau - Rocquencourt - BP 105 - 78153 Le Chesnay Cedex (France)

Éditeur

INRIA - Domaine de Voluceau - Rocquencourt, BP 105 - 78153 Le Chesnay Cedex (France)

http://www.inria.fr

ISSN 0249-6399 